\title{
Core-shell superparamagnetic nanoparticles with interesting properties as contrast agents for MRI
}

\author{
A. Carvalho ${ }^{\text {a, }}$, I. Domingues ${ }^{b}$, M. Clara Gonçalves ${ }^{c, d}$ \\ a CENIMAT- I3N/DCM/FCT/UNL, Faculdade de Ciências e Tecnologia da Universidade Nova de Lisboa, Campus da Caparica, 2829-516 Caparica, Portugal \\ ${ }^{\mathrm{b}}$ Department of Biology and CESAM, Universidade de Aveiro Campus Universitário de Santiago, 3810-193 Aveiro, Portugal \\ ${ }^{\mathrm{c}}$ Departamento de Engenharia Química, Instituto Superior Técnico, Universidade de Lisboa, Av. Rovisco Pais, 1049-001 Lisboa, Portugal \\ d Centro de Química Estrutural, Instituto Superior Técnico, Universidade de Lisboa, Av. Rovisco Pais, 1049-001 Lisboa, Portugal
}

\section{H I G H L I G H T S}

- Core-shell nanoparticles with iron oxide cores and different coatings were obtained.

- The new nanosystems are efficient as negative contrast agent for MRI.

- The efficiency is discussed relating to the different coatings properties.

- Contrast enhancement is quantified in phantoms MRI and validated in an animal model.

- The new core-shell systems have the possibility of bio-conjugation.

\section{A R T I C L E I N F O}

\section{Article history:}

Received 25 September 2014

Received in revised form

8 June 2015

Accepted 13 October 2015

Available online 6 November 2015

\section{Keywords:}

Nanostructures

Nuclear Magnetic Resonance (NMR)

Magnetic materials

Microporous materials

Sol-gel growth

\begin{abstract}
A B S T R A C T
Core-shell nanoparticles (NPs) formed by superparamagnetic iron oxide NPs (SPIONs) coated with inorganic or organically modified (ORMOSIL) sol gel silica exhibited promising properties as negative contrast agents (CA) for MRI applications.

The potentiality of these new core-shell NPs as negative CA for MRI is demonstrated and quantified.

The longitudinal and transverse relaxivities of NPs with three different coating compositions were studied at a $7 \mathrm{~T}$ magnetic field: silica (TEOS), (3-aminopropyl) triethoxysilane (APTES) and (3glycidoxypropyl) methyldiethoxysilane (GPTMS).

Clearly, it was found that the core-shell NPs efficiency as CA was strongly depend on the SPIONs coating.

All the three core-shell NPs studied presented a very small effect on the longitudinal relaxation time but a pronounced one on the transverse relaxation time, leading to a very high transverse longitudinal relaxivities ratio, decisive for their efficiency as negative CA for MRI.

The effect of the core-shell NPs on the MRI contrast enhancement is obtained and quantified in a set of MRI of agar phantoms obtained at $7 \mathrm{~T}$ magnetic field and with a imaging gradient field of $1.6 \mathrm{~T} / \mathrm{m}$. The core-shell NPs were tested in Zebra-fish (Danio rerio) animal model. Zebra-fish MRI were obtained with animals injected with the three core-shell NPs and the contrast enhancement validated.
\end{abstract}

(C) 2015 Elsevier B.V. All rights reserved.

\section{Introduction}

Magnetic resonance imaging (MRI) is considered the most important development in medical diagnostic since the discovery of X-rays more than 100 years ago. This classification is due, mainly, to the high spatial resolution and to the absence of ionizing

\footnotetext{
* Corresponding author.

E-mail addresses: alex.carvalho@netcabo.pt, amfc.carvalho@fct.unl.pt (A. Carvalho).
}

radiation during imaging process.

In other to achieve better image quality, along with faster and safer diagnostics, MRI as evolve to high magnetic fields [1,2]. When the contrast among neighbouring tissues is not sufficient for diagnostic purposes, the use of chemical contrast agents (CA) may be employed to enhance tissue contrast, by shorting the tissue/ medium longitudinal and/or transverse relaxation times.

During the last decades, the huge knowledge acquired on the molecular/cellular process of disease has driven the necessity to 
new and more efficient CA [3-6]. The CA are based on paramagnetic compounds or particles [7] and superparamagnetic NPs $[8,9]$. Among the last, superparamagnetic iron oxide nanoparticles (SPIONs) emerge as the most promising [10]. Recently a comprehensive review on SPIONs design and applications for MRI was published [11]. The interest in superparamagnetic NPs and, particularly, SPIONs in biomedicine far exceeds the CA application for MRI [12]; a new imaging modality based exclusively on the SPIONs detection, the Magnetic Particle Imaging (MPI) [13], is attracting a lot of expectation.

Despite the relevance of SPIONs in theranosis, SPIONs need to be coated with a biocompatible material able to: i) protect them against agglomeration, ii) hinder their surface activity, iii) provide chemical handles for further bioconjugation with drug molecules, targeting ligands, etc. and iv) limit non-specific SPIONs-cell interactions.

As for the SPIONs application here presented, the use as CA for MRI, it is known that the coating it-self is important on the enhancement of image contrast [14], this aspect will be explored in this work.

Silica plays a crucial role in the structural integrity of nails, hair, skin, overall collagen synthesis, bone mineralization and health and in reducing metal accumulation in Alzheimer's disease, immune system health, and reduction of the risk for atherosclerosis [15-17]. Amorphous silica has been part of human dietary either as component in medical clay or as a food additive (labelledE551) whereby it could enter the human body via the gasto-intestinal tract. Due to its extremely high biocompatibility and biodegradability, $\mathrm{SiO}_{2}$ arises as special material when it comes to SPIONs coating, $[18,19]$. Silica-coating NPs offer high biocompatibility, high hydrophilicity, and enormous versatility for surface modification with a high payload capacity, a prolonged blood circulation time, giving rise to wide range of biomedical and pharmaceutical applications, namely, gene or drug deliver, different imaging modalities like fluorescence or actively targeted nanoprobes [20-25]. Furthermore, silica-coating increases SPIONs dispersion in aqueous liquid media and biologic fluids, prolonging blood circulation time, by screening the magnetic dipole attraction among the superparamagnetic cores, along with providing an hydrophilic surface character.

The development of multifunctional silica-based platforms for use in nanomedicine has reached a great deal of interest in the scientific community [26-30].

ORMOSIL [31], has even more versatile properties than inorganic silica; the presence of non-hydrolysable organic groups allows an easier chemical conjugation/decoration of biomolecules at the ORMOSIL surface and/or the load with either hydrophilic or hydrophobic drugs/dyes, besides a tunable wettability of the synthesized material by a judicious choice of the ratio of hydrophilic to hydrophobic monomers can be achieved. ORMOSIL NPs conjugated with fluorophores and targeting ligands have been used in optical imaging of tumour cells in vitro [32]. The organic groups bonded to a siloxane skeleton besides retarding the sol- gel process of the organoalkoxysilane acts as fillers in the silica network, conferring some degree of flexibility to the otherwise rigid silica matrix, changing the porosity profile besides modifying the NPs colloidal chemistry. ORMOSIL NPs have also been tested for potential use in photodynamic therapy, and as targeted optical probes for imaging of human cervix epitheloid carcinoma cells in vitro experiments [33] and have been tested in animal models, namely in Zebra-fish.

Zebra-fish model presents many characteristics that make it well suited for studies of nanomedicine, namely: a short generation time ( 3 months), large hatch size (more than 200 eggs), rapid embryogenesis (most of the organs developed in $96 \mathrm{~h}$ post- fertilization) [34,35]. Zebra-fish model will be used in this work.

In the present work, three different silica/ORMOSIL compositions are studied as coatings for SPIONs to be used as negative CA for MRI, and promising candidates for cellular and molecular imaging.

As stated before, clinical MRI as evolve to high magnetic fields $[1,2]$, and this is the reason for the use of $7 \mathrm{~T}$ magnetic field in the present work.

\section{Materials and methods}

\subsection{Materials}

Iron (II) chloride tetrahydrate $\left(\mathrm{FeCl}_{2}\right)$, iron (III) chloride hexahydrate $\left(\mathrm{FeCl}_{3}\right)$ and hydrochloric acid $(\mathrm{HCl})$, (3-Aminopropyl) triethoxysilane (APTES), aqueous ammonia solution $\left(\mathrm{NH}_{4} \mathrm{OH}, 28 \%\right)$ and (3-Glycidoxypropyl) methyldiethoxysilane (GPTMS) were purchased from Sigma-Aldrich; tetraethylorthosilicate (TEOS) was purchased from Merck and ethanol $\left(\mathrm{CH}_{3} \mathrm{CH}_{2} \mathrm{OH}\right)$ from Panreac.

\subsection{Synthesis of the core-shell NPs}

SPIONs were synthesized by slight modification of the Berger method as reported in [36], where a strong alkali is added to an aqueous solution of iron(II) and iron(III), allowing the coprecipitation of the corresponding hydroxides, followed by fast aging and size control of iron oxides promoted by sonication.

Briefly, iron (II and III) chloride solutions were prepared in $\mathrm{HCl}$ medium (2M): $0.3975 \mathrm{~g}$ of iron (II) chloride tetrahydrate $\left(\mathrm{FeCl}_{2} 4 \mathrm{H}_{2} \mathrm{O}\right)$ was dissolved in $1 \mathrm{~mL} \mathrm{HCl}$ (solution $\mathrm{A}$ ) and $0.2702 \mathrm{~g}$ of iron (III) chloride hexahydrate $\left(\mathrm{FeCl}_{3} 6 \mathrm{H}_{2} \mathrm{O}\right.$ ) in $4 \mathrm{~mL} \mathrm{HCl}$ (solution $\mathrm{B}$ ). The two iron solutions were mixed together $(A+B)$, under sonication and $50 \mathrm{~mL}$ of aqueous ammonia solution $(0.7 \mathrm{M})$ were added drop wise to $(A+B)$ solution, under sonication, allowing SPIONs to precipitate. With the help of a magnet, the supernatant was discarded and SPIONs were washed four times with ethanol to remove excess reagents, and then left dried at $40{ }^{\circ} \mathrm{C}$ for $24 \mathrm{~h}$. On the day before SPIONs coating, SPIONs were weighted, dissolved in ethanol and left to settle overnight in suspension, discarding the deposited agglomerates.

SPIONs were silica/ORMOSIL coated in situ by the LaMer nucleation method [37,38].

The LaMer method, supported on the protocols described in $[24,39,40]$, allowed the synthesis of NPs (roughly $100 \mathrm{~nm}$ in diameter), at room temperature in less than $1 \mathrm{~h}$. SPIONs were used as seeds for inorganic and hybrid silica nucleation, driven to cor$\mathrm{e}$-shell nanostructures. In typical sol-gel process alkoxide precursors (APTES, GPTMS and TEOS) undergo hydrolysis to form silanol groups. In a first step, the silanols link to $-\mathrm{OH}$ or surface oxygens from SPIONs, and in a second step, silanols react with other silanols or with siloxanes, allowing the silica or ORMOSIL coating growth. Shell thickness is dependent upon the number of nucleus available (SPIONs concentration) and upon the precursors' nature. It is well known the effect of steric hindrance on hydrolysis and condensation kinetcs of sol-gel reactions.

SPIONs ( $47.5 \mathrm{mg}$ ) were added to a solution (named C) composed by $44.7 \mathrm{~mL}$ of ethanol, $2.25 \mathrm{~mL}$ of bidistilled water and $1.15 \mathrm{~mL}$ of ammonia. The resulting solution named solution (D) is magnetically stirred until temperature reached $55^{\circ} \mathrm{C}$. A solution with $1.9 \mathrm{~mL}$ of total precursor (solution $\mathrm{E}$ ) is rapidly added to solution $\mathrm{D}$ and ultrasonicated for $10 \mathrm{~min}$. Then, this mixture is stirred at $55{ }^{\circ} \mathrm{C}$ during $2 \mathrm{~h}$. Synthesized NPs are then isolated by centrifugation $(8000 \mathrm{rpm})$ and four times washed with bidistiled water and ethanol to remove alcohol, ammonia and eventual unreacted chemicals. NPs are further heated at $40{ }^{\circ} \mathrm{C}$ for $24 \mathrm{~h}$.

The obtained core-shell NPs will be named in the following for 
simplicity, by the coating: core-shell NPs-TEOS, NPs-GPTMS and NPs-APTES, referring to the precursors used: tetraethylorthosilicate (TEOS), (3-glycidoxypropyl) methyldiethoxysilane (GPTMS) and (3aminopropyl) triethoxysilane (APTES).

For the core-shell NPs-TEOS the only precursor used was TEOS, for the core-shell NPs-GPTMS solution E was composed of 1:9 of GPTMS and TEOS and for the core-shell NPs-APTES solution E was 1:9 of APTES and TEOS in molar ratio.

\subsection{Core-shell NPs characterization}

Particle morphology features static diameter and static diameter distribution were observed by transmission electron microscopy (TEM). TEM micrographs were obtained using a Hitachi H-8100, using an applied tension of $200 \mathrm{kV}$. To analyse the samples, a droplet of the suspension was deposited on the copper grid and dried at room temperature.

Statistic diameters measurements were performed with Adobe Photoshop CS5 on TEM images. The presented diameter is the mean of 150 core-shell NPs measurement and the standard deviation is roughly $10 \%$.

Fourier transform infrared (FTIR) spectra were obtained using a Nicolet 5700 in transmission mode in the medium infrared range. The ORMOSIL NPs were finely ground and mixed with potassium bromide, and then pressed into a disc. FTIR was used to confirm the organic nature of the ORMOSIL NPs structure.

The total iron content of core-shell NPs was determined by Inductively Coupled Plasma-Atomic Emission Spectrometry (ICPAES) with a $15 \%$ uncertainty.

\subsection{Nuclear magnetic relaxivities}

The efficiency of a CA for MRI is measured by its longitudinal $\left(r_{1}\right)$ and transverse $\left(r_{2}\right)$ relaxivities and their ratio. The longitudinal and transverse relaxivities values measures the capacity of the CA to alter the tissue/medium longitudinal and transverse relaxation times, respectively. Actually, a CA changes both the tissue/medium relaxation times, but if the main effect is on shortening the longitudinal relaxation time the CA is classified as positive, on the other hand, if the main effect is decreasing the tissue/medium transverse relaxation time, the CA is labelled as negative. An efficient negative CA presents a high ratio $r_{2} / r_{1}$ meaning that the tissue/medium $T_{2}$ is shorten but $T_{1}$ is little affected.

To obtain the $r_{1}$ and $r_{2}$ of the three core-shell NPs an aqueous agar solution was prepared $0.5 \%$ in weight which simulates the magnetic relaxation properties of human tissues [41]. With this solution, for each core-shell NPs, a set of solutions was prepared, with iron (I and II) concentrations ranging from 0.01 to $0.87 \mathrm{mM}$ the solutions were kept in standard $5 \mathrm{~mm}$ NMR tubes. The relaxation times $T_{1}$ and $T_{2}$ were measured at $25{ }^{\circ} \mathrm{C}$ in a Bruker Avance III NMR spectrometer, in a $7 \mathrm{~T}$ magnetic field ( $300 \mathrm{MHz}$ for proton) by high resolution spectroscopy. For the $T_{1}$ measurement an usual inversion-recovery pulse sequence was used with a $T_{R}$ of $15 \mathrm{~s}$. For $T_{1}$ measurement 16 different inversion times were considered. The $T_{2}$ was obtained with the Carr-Purcell-Meiboom-Gill sequence with the precaution that the measurement time (two echo-time times the number of acquired echos) always defines a time interval of about 10 times $T_{2}\left(2 \tau_{\text {echo }} N_{\text {echo }} \geq 10 T_{2}\right)$. The longitudinal $\left(1 / T_{1}\right)$ and transverse $\left(1 / T_{2}\right)$ relaxation rates follows:

$\frac{1}{T_{i}}=\frac{1}{T_{i_{o}}}+r_{i}[\mathrm{Fe}]$

where $\mathrm{i}=1,2$ represent longitudinal and transverse respectively, $T_{i_{o}}$ the relaxation time longitudinal or transverse in the absence of the core-shell NPs, $r_{i}$ the longitudinal or transverse relaxivities and [ $\left.\mathrm{Fe}\right]$ the iron concentration [42].

\subsection{Phantom magnetic resonance micro-imaging}

Three phantom were constructed. Each one consisted of two $5 \mathrm{~mm}$ standard NMR tubes: one with aqueous agar solution $0.5 \%$ in weight (blank) and the other with the core-shell NPs in the aqueous agar solution. The total iron (II and III) concentration in these core-shell NPs in the aqueous solution was: $0.17 \mathrm{mM}$ for the core-shell NPs-TEOS, $0.15 \mathrm{mM}$ for the core-shell NPs-GPTMS and $0.17 \mathrm{mM}$ for the core-shell NPs-APTES, to allow comparison.

Magnetic resonance images were obtained in a Bruker Avance III NMR spectrometer, in a $7 \mathrm{~T}$ magnetic field equipped with a imaging magnetic gradient field of $1.6 \mathrm{~T} / \mathrm{m}$, allowing a micro-image study. MRI of $1 \mathrm{~mm}$ axial slices of the phantom were collected, with a FOV of $1.16 \times 1.16 \mathrm{~cm}^{2}$ using a spin-echo pulse sequence and four images obtained with echo-times of $15,30,45$ and $60 \mathrm{~ms}$.

\subsection{Zebra-fish model}

For animal model study the zebra-fish (Danio rerio) was used, following recent works where non-mammalian vertebral animals are used as models in nanomedicine research [43]. Danio rerio were obtained from the facility established at the Department of Biology, University of Aveiro, Portugal. Adult Danio rerio are maintained in carbon-filtered water complemented with salt Instant Ocean Synthetic Sea Salt (Spectrum Brands, USA), at $27.0 \pm 1{ }^{\circ} \mathrm{C}$ and under a 14:10 h light:dark photoperiod cycle. Conductivity is kept at $750 \pm 50 \mu \mathrm{S} / \mathrm{cm}, \mathrm{pH}$ at $7.5 \pm 0.5$ and the saturation of dissolved oxygen $>95 \%$. Zebra-fish adults were fed twice daily with commercially available artificial diet (ZM 400 Granular, ZM systems, Hampshire, UK) and brine shrimp.

Zebra-fish were anaesthetized, by immersion in tricaine methanesulfonate (MS222, from Sigma-Aldrich), and transferred to custom-made agarose gel molds, to hold them in place. They were immediately micro injected intraperitoneal in the abdominal cavity with IM 300 Microinjector from Narishige [44]. The administrated dose, in the absence of reference values for these animals, was $13 \mathrm{mg} / \mathrm{kg}$. For each core-shell NPs obtained, two zebra-fishes groups composed of three elements were sacrificed: one group $30 \mathrm{~min}$ after injection and the other $48 \mathrm{~h}$ after injection. Also a nonexposed control group of zebra-fishes were sacrificed at the same biological time. Animals were euthanized with an overdose of MS222 and preserved in Dietrich's fixative.

For the MRI study the zebra-fishes were removed from the Dietrich's fixative and immersed in perfluoropolyether oil. MRI of $1 \mathrm{~mm}$ slice of the zebra-fishes were collected in axial, sagittal and coronal directions with a FOV of $4 \times 4 \mathrm{~cm}^{2}$ with a spin-echo pulse sequence ( $\mathrm{T}_{R}$ of $2.5 \mathrm{~s}$ and a four $\mathrm{T}_{E}$ in the range $7.0-28.0 \mathrm{~ms}$ ) with 4 excitations. Recent studies in Drosophila indicates that this core-shell NPs are biocompatible and not toxic to whole organisms [45].

\section{Results}

\subsection{Core-shell NPS}

SPIONs have been synthesized and characterized as in a previous work [36]. Quasi-spherical, ultra small ( $\sim 6 \mathrm{~nm})$, narrow size distribution SPIONs $\left(5 \% \mathrm{Fe}_{3} \mathrm{O}_{4}\right.$ and $\left.95 \% \gamma-\mathrm{Fe}_{2} \mathrm{O}_{3}\right)$ with superparamagnetic behaviour are the core of the core-shell NPs obtained. SPIONs have been coated with three different silica-based materials: inorganic silica (core-shell NPs-TEOS), amino- 
Table 1

Core-shell NPs mean static diameters obtained by TEM measurements.

\begin{tabular}{llll}
\hline & NPs-APTES & NPs-GPTMS & NPs-TEOS \\
\hline $\mathrm{d}(\mathrm{nm})$ & $96.6 \pm 9.6$ & $22.0 \pm 2.2$ & $66.6 \pm 6.6$ \\
\hline
\end{tabular}

functionalized silica (core-shell NPs-APTES) and (3glycidoxypropyl) methyldiethoxysilane functionalized silica (core-shell NPs-GPTMS).

Table 1 shows the core-shell NPs mean static diameter and Fig. 1 illustrates core-shell NPs-TEOS, NPs-GPTMS and NPs-APTES as an example of the NPs obtained.

\subsection{Nuclear magnetic relaxivities}

The longitudinal and transverse relaxivities were obtained from the fit of Eq. (1) to the experimental longitudinal and transverse relaxation rates. The experimental results and the fitting of Eq. (1) are presented in Fig. 2 and the obtained $r_{1}, r_{2}$ are shown in Table 2.

All the core-shell NPs present a negligible effect on $T_{1}$, and a large effect on $T_{2}$. This result makes their use as negative CA for MRI promising. The same negligible effect $T_{1}$ although in a smaller scale was reported for a silica coated SPIONs [14].

Core-shell NPs-APTES exhibited a distinct finger print as can be observed in Fig. 2 where transverse relaxation rate $v s$ iron is plotted.

A negative CA for MRI efficiency is measured by the $r_{2}$ value and the $r_{2} / r_{1}$ ratio. Although higher values for $r_{2}$ have been reported [46], in the present work the ratio $r_{2} / r_{1}$ is outstanding high, due to the extremely low $r_{1}$ values. For comparison, in Table 2 the $r_{1}, r_{2}$ and $r_{2} / r_{1}$ for a commercial CA based on SPIONs (obtained also at $7 \mathrm{~T}$ ) are presented [47]. To the best of our knowledge the $r_{2} / r_{1}$ ratios presented here for the first time, in particular at 7T, are the highest observed yet. Adding to this promising result, our core-shell NPs, in particular the core-shell NPs-APTES, is the first choice for gene conjugation opening the door to cellular or molecular imaging [24].

\subsection{Phantom MRI analysis}

The obtained MRI of the three phantoms are presented in Fig. 3. Both the core-shell NPs-TEOS and NPs-APTES presented a good contrast. However, for the fourth echo, the core-shell NPs-APTES presented a higher noise level. It can be observed that the worse contrast is obtained for the core-shell NPs GPTMS, Fig. 4.

The contrast to noise ratio for the phantom images was calculated by the following procedure: three $0.1 \mathrm{~cm}^{2}$ regions of interest (ROI) were considered, a first in the core-shell NPs region (NPs) a second in the agar region (agar) and a third in the background region $(\mathrm{N})$. The signal intensity was calculated for the three regions, $I_{\text {agar }}, I_{N P s}$ and $I_{N}$. The contrast, agar core-shell NPs to noise ratio was calculated as:
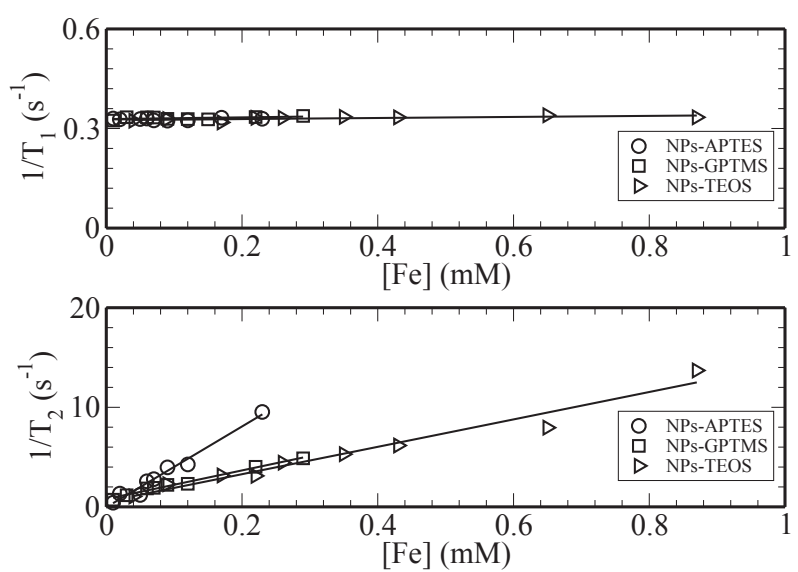

Fig. 2. Longitudinal relaxation rate $\left(1 / T_{1}\right)$ vs iron oxide content and transverse relaxation rate $\left(1 / T_{2}\right)$ vs iron oxide content for the three core-shell NPs.

Table 2

Longitudinal and transverse relaxivities and $r_{2} / r_{1}$ ratio for the core-shell NPs measured at a $7 \mathrm{~T}$ magnetic field and for Endorem a commercial CA [47] in the same conditions.

\begin{tabular}{llcc}
\hline & $\mathrm{r}_{1}(\mathrm{mMs})^{-1}$ & $\mathrm{r}_{2}(\mathrm{mMs})^{-1}$ & $\mathrm{r}_{2} / \mathrm{r}_{1}$ \\
\hline NPs-APTES & 0.006 & 40.6 & 6767 \\
NPs-GPTMS & 0.026 & 14.4 & 554 \\
NPs-TEOS & 0.016 & 13.8 & 863 \\
Endorem (Guerbert) & 2.2 & 182 & 82.7 \\
\hline
\end{tabular}

$C / N=\frac{\left|I_{\text {agar }}-I_{N P S}\right|}{I_{N}}$

The $C / N$ result for the core-shell NPs-GPTMS phantom (the smaller obtained NPs) and for the core-shell NPs-APTES (the larger obtained NPs) phantom are presented in Fig. 4. A superior performance of the core-shell NPs-APTES, at least for the shorter echotimes, was observed.

\subsection{Zebra-fish MRI}

No fish mortality was observed after injection and zebra-fish behaviour was not affected during the experiment period $(48 \mathrm{~h})$. The MRI contrast obtained for the zebra-fishes treated with the different core-shell NPs presented clear differences.

Control fishes (not injected with any core-shell NPs) originated greyish images and with low capacity of organ discrimination in the abdominal region, Fig. 5.

MRI obtained of zebra-fishes injected with core-shell NPsAPTES and core-shell NPs-TEOS show high definition of anatomic structures.

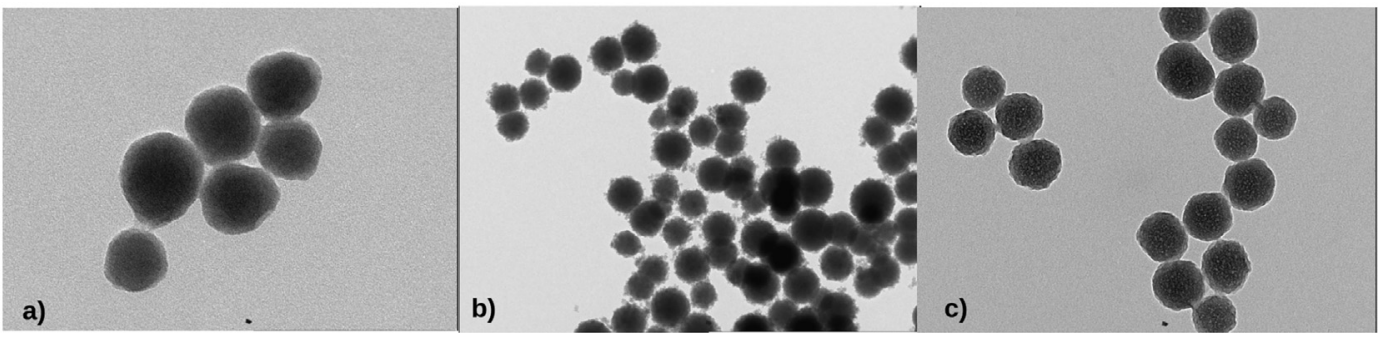

Fig. 1. Transmission electronic microscopy of a) core-shell NPs-TEOS b) core-shell NPs-GPTMS and c) core-shell NPs-APTES. Amplification $10^{5}$. 


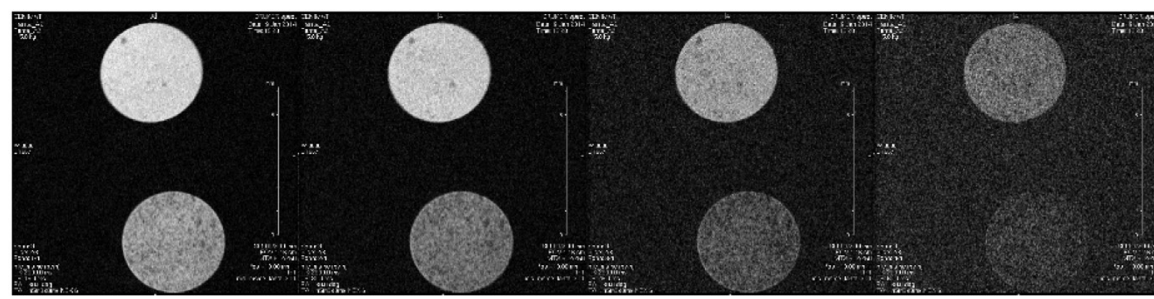

Agar

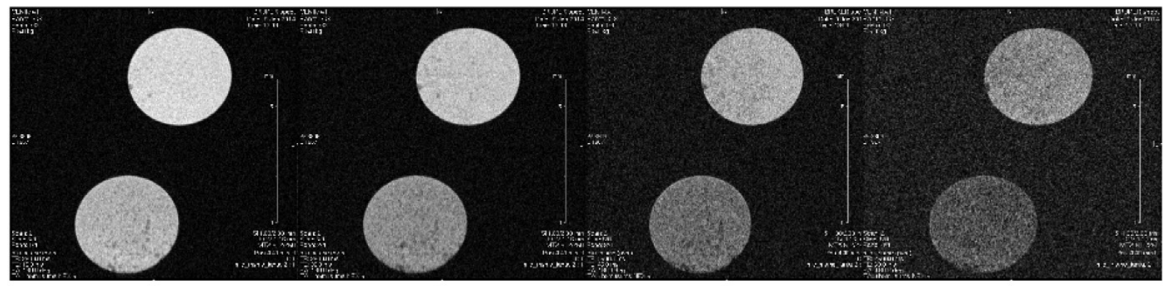

NPs-APTES

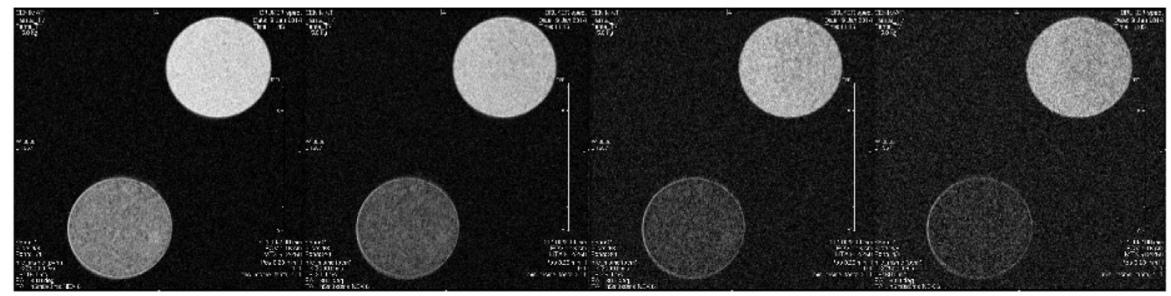

Agar

NPs-GPTMS

Agar

NPs-TEOS

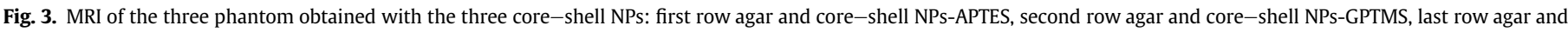
core-shell NPs-TEOS. The MRI were obtained for echo-time 15, 30, 45 and $60 \mathrm{~ms}$.

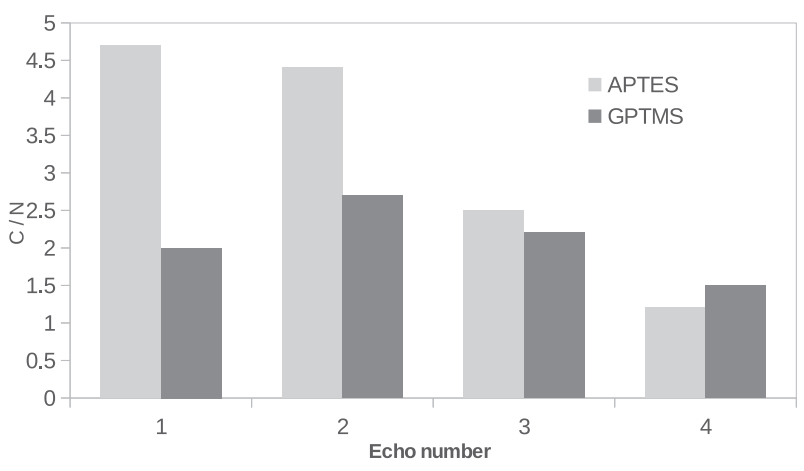

Fig. 4. Contrast to noise ratio for images of the two phantom agar and core-shell NPsAPTES and the agar and core-shell NPs-GPTMS obtained for the four echos corresponding 15, 30, 45 and $60 \mathrm{~ms}$ echo-times, respectively.

In Fig. 6, longitudinal and axial slices of zebra-fish injected with core-shell NPs-APTES are shown. In the longitudinal plans muscle fibbers can be observed in an individualized way and with a high level of detail that could not be observed in control zebra-fish images, Fig. 5. In the axial plans the abdominal content also presents more contrast and detail than the one observed in the control zebra-fishes MRI.

MRI of zebra-fishes injected with core-shell NPs-GPTMS were also obtained although not presented for shortness. In axial images organs and structures also present a high contrast. In longitudinal slices the level of detail and differentiation of muscle fibbers do not seem so good being similar to the results obtained with the control zebra-fishes.

Fig. 7 presents MRI of zebra-fishes injected with core-shell NPsTEOS where a detail similar the ones injected with core-shell NPsAPTES was observed.

As for the time delay between the injection of the core-shell NPs and the zebra-fishes sacrifice (30 min and $48 \mathrm{~h}$ ) no observable difference in MRI contrast was reported.

\section{Discussion}

It is known [48], that the SPIONs relaxivities depends on the NPs magnetization and on the accessibility of water molecule to the SPIONs mean magnetic moment $\mu$. The SPIONs magnetic moment

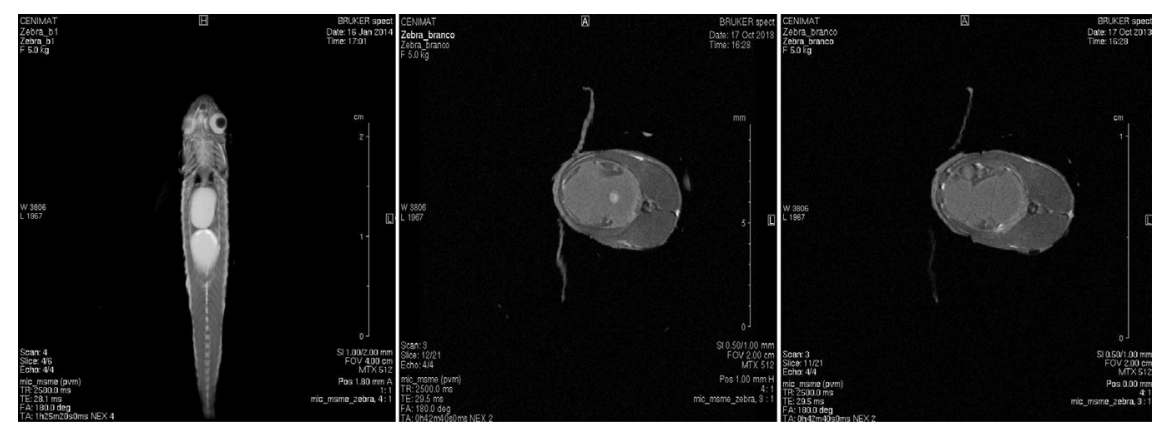

Fig. 5. MRI of the two zebra-fishes not injected with core-shell NPs. 

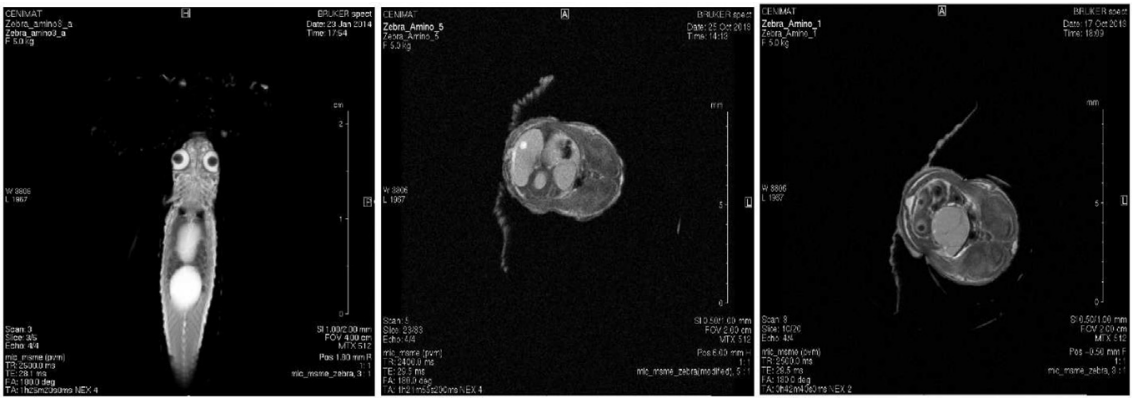

Fig. 6. MRI of longitudinal and axial slices of zebra-fishes injected with core-shell NPs-APTES
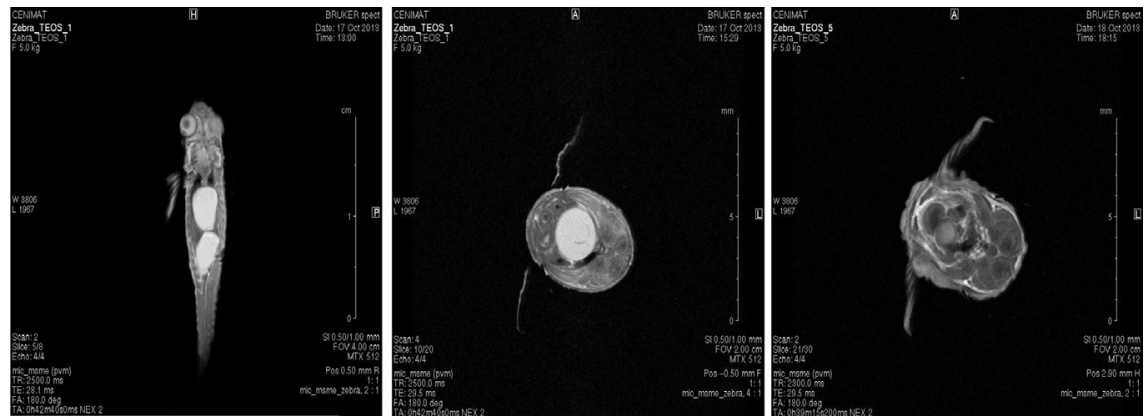

Fig. 7. MRI of two zebra-fishes injected with core-shell NPs-TEOS.

increases with the magnetic field up to saturation $\mu_{\text {sat }}$ following Langevin function $\mathrm{L}(\mathrm{x})$ :

$\mu=\mu_{\text {sat }} L(x)$

where $L(x)=1 / \tanh (x)-1 / x, x=\mu_{\text {sat }} B / K T, \mathrm{~K}$ is the Boltzmann constant and $T$ the temperature.

The different coatings, of the synthesized core-shell NPs gave the opportunity for analysing different accessibility of water molecules to these SPIONs magnetic moment.

The $r_{1}$ and $r_{2}$ of the core-shell NPs were obtained at a $7 \mathrm{~T}$ magnetic field, which has to be taken into account in direct values comparison. As referred, this high field measurement is important since clinical MRI is evolving to higher fields.

SPIONs coating composition is very important to the CA efficiency. The coating effect is being study by other researchers $[14,49]$ in a variety of compositions.

The negligible effect of our core-shell NPs on the longitudinal relaxation rate, may be related to the coating effect hindering the water access to the SPIONs magnetic moment. This effect is strongly related with the coating thickness, the thicker the coating the smaller $r_{1}$. The NPs-APTES shows the ticker coating radius exhibiting the smaller $r_{1}$. The presence of organic groups affects ORMOSIL coating's thickness for identical synthesis protocol. The replacement of TEOS by APTES, drive to an increase in core-shell diameter (relatively to silica NPs), while the replacement by GPTMS lead to a decrease. Due to both electronic and steric factors, the presence of the organic components bonded to a siloxane or to silica backbone, plays a role in hydrolysis and condensation rates. Steric factors exert the greatest effect on both rates. Hydrolyses and condensation rates are retarded by steric hindrance, being lowered the most by branched alkoxy groups, APTES and GPTMS in the present case. This is the reason why core-shell NPs-GPTMS have smaller diameter (for the same reaction time).
The SPIONs coating by silica or ORMOSIL didn't destroy the capacity of the SPIONs to create a inhomogeneous magnetic field that enhances $T_{2}$ relaxation. The core-shell NPs presented interesting transverse relaxivities for a high magnetic field $(7 \mathrm{~T})$. It is known that $r_{2}$ decreases with the magnetic field for values above roughly $0.2 \mathrm{~T}[50]$.

It is a fact that several SPIONs present higher $r_{2}$ values [14] but the NPs here studied have outstanding ratios $r_{2} / r_{1}$. Since all the synthesized NPs have the same superparamagnetic core, the observed differences in the ratio $r_{2} / r_{1}$ mus be assigned to the coating. And by coating effect we mean (i) coating thickness (ii) coating surface chemistry, (iii) coating porosity (pore size, pore size distribution, pore shape, and pore surface character-hydrophilic versus hydrophobic nature).

It is reported $[49,14]$ that the hydrophilicity of the SPIONs coating affect the obtained relaxivities. The different organic groups bonded to the siloxane skeleton provides different hydrophilicity to the obtained core-shell NPs and this reflects in the observed relaxivities.

Silica and ORMOSIL NPs porosity study has been performed by the authors and is reported elsewhere [51] it revels a correlation between the coating pore size and the ratio $r_{2} / r_{1}$ of the core shell NPs. Indeed the NPs-APTES presented a pore diameters that is roughly double that the other NPs pore diameters and the ratio $r_{2} / r_{1}$ for the NPs-APTES is much larger them the others, as can be observed in Table 2 . This fact directly relates to the accessibility of water molecule to the NPs superparamegnetic core.

From the MRI of phantoms prepared with the core-shell NPs a contrast enhancement for all the NPs was observed. The coating effect is translated into image contrast. The core-shell NPs-TEOS and NPs-APTES presented good contrast to noise ratio. For the short echo-time images, it is roughly, twice the contrast to noise ratio obtained for the core-shell NPs-GPTMS.

The test in zebra-fish animal model corroborates the above 
observations: the zebra-fishes injected with core-shell NPs-APTES and the ones injected with NPs-TEOS presented, in MRI, good anatomical detail, superior to the one shown in MRI of the control. The zebra-fishes injected with the core-shell NPs-GPTMS, no improvement in the anatomical detail was observed in comparison with the control group.

\section{Conclusions}

In this paper the potential of three new silica-based core-shell NPs as negative CA for MRI was demonstrated.

SPIONs coated with silica, (3-aminopropyl) trietoxysilane (APTES) and (3-glycidoxypropyl) methyldiethoxysilane (GPTMS) were synthesized with $\sim 100 \mathrm{~nm}$ in diameter. All the studied core-shell NPs exhibited a very high ratio $r_{2} / r_{1}>500$, for $7 \mathrm{~T}$ magnetic field, resulting from a decrease in the tissue/medium $T_{2}$ along with a minor effect on $T_{1}$. To the best of our knowledge the highest ratio ever reported for a SPIONs.

Furthermore, the $r_{2} / r_{1}$ ratio was observed to be strongly depend on the shell-composition.

A good MRI contrast enhancement was confirmed in a set of agar phantoms, obtained at $7 \mathrm{~T}$ magnetic field, with a imaging gradient field of $1.6 \mathrm{~T} / \mathrm{m}$. The contrast enhancement for the studied core-shell NPs; may be ordered as NPs-APTES > NPs-TEOS > NPsGPTMS.

Zebra-fish animal model was used to validate the MRI contrast enhancement along with NPs innocuous. No fish mortality was observed after injection or during the experimental period.

With these core-shell NPs it is possible to tune the relaxivities of the obtained CA but perhaps even more interesting the possibility of bio-conjugation with the core-shell NPs of ORMOSIL and in particular with the core-shell NPs-APTES opens the molecular or cellular imaging door.

\section{Acknowledgements}

A. Carvalho acknowledge financial support to: PEst-C/CTM/ LA0025/2013-14 and the Portuguese Nuclear Magnetic Resonance Network (PTNMR). I. Domingues kindly acknowledge the financial support SFRH/BPD/90521/2012. The authors gratefully acknowledge the technical support of V. Ferreira and J. F. Henriques from Aveiro University and T. Silva from I.S.T. in the manipulation of the zebra-fishes.

\section{References}

[1] H. Wada, M. Sekino, H. Ohsaki, T. Hisatsune, H. Ikehira, T. Kiyoshi, Prospect of high-field MRI, IEEE Trans. Appl. Supercond. 3 (2010) 3115-3122.

[2] J.H. Duyn, The future of ultra-high field MRI and fMRI for study of the human brain, Neuroimage 62 (2) (2012) 1241-1248.

[3] M.L. Matson, L.J. Wilson, Nanotechnology and MRI contrast enhancement, Fut. Med. Chem. 2 (3) (2010) 491-502.

[4] E. Terreno, D. Castelli, A. Viale, S. Aime, Challenges for molecular magnetic resonance imaging, Chem. Rev. 110 (5) (2011) 3019-3042.

[5] A.G. Roca, R. Costo, A.F. Rebolledo, S. Veintemillas-Verdaguer, P. Tartaj, T. Gonzalez-Carreño, C.J. Serna, Progress in preparation of magnetic nanoparticle for applications in biomedicine, J. Phys. D. Appl. Phys. 42 (2009), 224002 (11pp.).

[6] A.J.L. Villaraza, A. Bumb, M. Brechbiel, Macromolecules, dendrimers and nanomaterials in magnetic resonance imaging, Chem. Rev. 110 (5) (2010) 2921-2959.

[7] M. Norek, G.A. Pereira, C.F.G.C. Geraldes, A. Denkova, W. Zhou, J.A. Peters, NMR tranversal relaxivity of suspensions of lanthanide oxide nanoparticles, J. Phys. Chem. C. 111 (2007) 10240-10246.

[8] A. Carvalho, A. Taborda, Unusual longitudinal relaxation time behaviour of colloid solutions of superparamagnetic nanoparticles, Eur. Phy. J. Appl. Phys. 40 (2007) 265-268.

[9] A. Taborda, A. Carvalho, Superparamagnetic iron oxide nanoparticles-proton nuclear magnetic resonance dispersion curves, Eur. Phy. J. Appl. Phys. 43 (2008) 145-148.
[10] J.W.M. Bulte, In vivo MRI cell tracking: clinical studies, AJR 193 (2009) 314-325.

[11] R. Jin, B. Lin, D. Li, H. Ai, Superparamagnetic iron oxide nanoparticles for MR imaging and therapy: design considerations and clinical applications, Curr. Opin. Pharmacol. 18 (2014) 18-27.

[12] Q.A. Pankhurst, N.K.T. Thanh, S.K. Jones, J. Dobson, Progress in applications of magnetic nanoparticles in biomedicine, J. Phys. D. Appl. Phys. 42 (2009), 224001 (15pp).

[13] B. Gleich, J. Weizenecker, Tomographic imaging using the nonlinear response of magnetic particles, Nature 435 (2005) 1214-1217.

[14] F. Ye, S. Laurent, A. Fornara, L. Astolfi, J. Qin, A. Roch, A. Martini, M.S. Toprak, R.N. Muller, M. Muhammed, Uniform mesoporous silica coated iron oxide nanoparticles as a highly efficient nontoxic MRI T2 contrast agent with tunable proton relaxivities, Contrast Media Mol. Imaging 7 (2012) 460-468.

[15] Keith R. Martin, in: K.O. Roland (Ed.), Interrelations between Essential Meta Ions and Human Diseases Metal Ions in Life Sciences, Ed. Sigel, Astrid, Sigel, Helmut, Sigel, vol. 13, Springer, 2013, pp. 451-473.

[16] C.T. Price, J.R. Langford, F.A. Liporace, Essential nutrients for bone health and a review of their availability in the average North American diet, Open Orthop. J. 6 (2012) 143-149.

[17] R. Jugdaohsingh, Silicon and bone health, J. Nutr. Health Aging 11 (2) (2007) 99-110.

[18] J.L. Campell, J. Arora, S.F. Cowell, A. Garg, P. Eu, S.K. Bhargava, V. Bansal, Quasicubic magnetite/silica core-shell nanoparticles as enhanced MRI contrast agents for cancer imaging, PLoS One 6 (7) (2011) e21857, http://dx.doi.org/ 10.1371/journal.pone.0021857.

[19] T. Ahmad, I. Rhee, S. Hong, Y. Chang, J. Lee, Silica-coated iron-oxide nanoparticles synthesized as a $T_{2}$ contrast agent for magnetic resonance imaging by using the reverse micelle method, J. Korean Phys. Soc. 57 (6) (2010) 1545-1549.

[20] D.J. Bharali, I. Klejbor, E.K. Stachowiak, P. Dutta, I. Roy, Organically modified silica nanoparticles: a nonviral vector in vivo gene delivery and expression in the brain, Proc. Natl. Acd. Sci. U. S. A. 102 (2005) 11539-11544.

[21] P. Couleaud, V. Morosini, C. Frochot, S. Richeter, L. Raehm, J.O. Durand, Silicabased nanoparticles for photodynamic therapy applications, Nanoscale 2 (2010) 1083-1095.

[22] L. Wang, W. Zhao, W. Tan, Bioconjugated silica nanoparticles: development and applications, Nano Res. 1 (2008) 99-115.

[23] S. Dash, S. Mishra, S. Patel, B.K. Mishra, Organically modified silica: synthesis and applications due to its surface interaction with organic molecules, Adv. Colloid Interfaces Sci. 140 (2008) 77-84.

[24] R. Colaço, M.C. Gonçalves, L. Fortes, L.M.D. Gonçalves, A. Almeida M.B. Martins, Preparation and chemical characterization of eco-friendly ORMOSIL nanoparticles of potential application in DNA gene therapy, Curr. Nanosci. 9 (1) (2013) 168-172.

[25] L.M. Fortes, Y. Li, R. Réfega, M.C. Gonçalves, Up-conversion in rare earth-doped silica hollow spheres, Opt. Mater. 34 (8) (2012) 1440-1446.

[26] P. Tallury, A. Malhotra, L.M. Byrne, S. Santra, Nanobioimaging and sensing of infectious diseases, Adv. Drug Deliv. Rev. 62 (2010) 424-437.

[27] S. Jiang, M.K. Gnanasammandhan, Y. Zhang, Optical imaging-guided cancer therapy with fluorescent nanoparticles, Interface 7 (2010) 3-18.

[28] A. Elsayed, M. Al-Remawi, I. Maghrabi, M. Hamaidi, N. Jaber, Development of insulin loaded mesoporous silica injectable particles layered by chitosan as a controlled release delivery system, Int. J. Pharm. 461 (2014) 448-458.

[29] S.Y. Lee, J. Lee, H.S. Lee, J.H. Chang, Rapid pathogen detection with bacterialassembled magnetic mesoporous silica, Biosens. Bioelectron. 53 (2014) $123-128$.

[30] X. Wu, M. Wu, J.X. Zhao, Recent development of silica nanoparticles as delivery vectors for cancer imaging and therapy, Nanomedicine Nanotechnol. Biol. Med. 10 (2014) 297-312.

[31] R.K. Sharma, S. Das, A. Maitra, Surface modified ormosil nanoparticles, J. Colloid Interface Sci. 277 (2004) 342-346.

[32] S. Santra, H. Yang, D. Dutta, J.T. Stanley, P.H. Holloway, TAT conjugated, FITC dopied silica nanoparticles for bioimaging applications, Chem. Comm. 24 (2004) 2810-2811.

[33] S. Kim, T.Y. Ohulchansky, H.E. Pudavar, R.K. Pandey, P.N. Prasad, Organically modified silica nanoparticles co-encapsulating photosensitizing drug and aggregation-enhanced two-photon absorbing fluorescent dye aggregates two-photon photodynamic therapy, J. Am. Soc. 129 (2007) 2669-2675.

[34] C.B. Kimmel, W.W. Ballard, S.R. Kimmel, B. Ulmann, T.F. Schiling, Stages of embryonic development of zebra-fish, Dev. Dyn. 203 (1995) 253-310.

[35] R. Dahm, R. Geisler, Learning from small fly: the zebra-fish as a genetic model organism for aquaculture fish species, Mar. Biotechnol. 8 (2006) 329-345.

[36] M.C. Gonçalves, L.M. Fortes, A.R. Pimenta, J.C.G. Pereira, R.M. Almeida, M.D. Carvalho, L.P. Ferreira, M.M. Cruz, M. Godinho, Silica/ORMOSIL magnetic core-shell nanoparticles, Curr. Nanosci. 9 (2013) 599-608.

[37] V.K. LaMer, R.H. Dinegar, Theory, production and mechanism of formation of monodispersed hydrosols, J. Am. Chem. Soc. 72 (11) (1950) 4847-4848.

[38] Y. Huang, J.E. Pemberton, Synthesis of uniform, spherical sub-100nm silica particles using a conceptual modification of the classic LaMer model, Colloids Surfaces A Physicochem. Eng. Aspects 360 (2010) 175-183.

[39] A. Arkhireeva, J.N. Hay, Synthesis of sub-200 nm silsesquioxane particles using a modified stober sol-gel route, J. Mater. Chem. 13 (2003) 3122-3127.

[40] P.A. Buining, L.M. Liz-Marzan, A.P. Philipse, A simple preparation of small, smooth silica spheres in a seed alcosol for stober synthesis, J. Colloid Interface 
Sci. 179 (1996) 318-321.

[41] R. Mathur-De Vre, R. Grimee, F. Parmentier, J. Binet, The use of agar gel as a basic reference material for calibrating relaxation times and imaging parameters, Magn. Res. Med. 2 (2) (1985) 176-179.

[42] E. Merbach, E. Tóth, The Chemistry of Contrast Agents in Medical Magnetic Resonance Imaging, Wiley, 2001, p. 46.

[43] M. Giannaccini, A. Cuschieri, L. Dente, V. Raffa, Non-mammalian vertebrate embryos as models in nanomedicine, Nanomedicine Nanotechnol. Biol. Med. 10 (2014) 703-719.

[44] M.D. Kinkel, S.C. Eames, L.H. Philipson, V.E. Prince, Intraperitoneal injection into adult Zebrafish, J. Vis. Exp. 42 (2010) e2126, http://dx.doi.org/10.3791/ 2126 (2010).

[45] F. Barandeh, P. Nguyen, R. Kumar, G.J. Iacobucci, M.L. Kuznicki, A. Kosterman, E.J. Bergey, P.N. Prasad, S. Gunawardena, Organically modified silica nanoparticles are biocompatible and can Be targeted to neurons in vivo, PLoS One 7 (1) (2012) e29424, http://dx.doi.org/10.1371/journal.pone.0029424.

[46] Y. Gossuin, P. Gillis, A. Hocq, Q.L. Vuong, A. Roch, Magnetic resonance relaxation properties of superparamagnetic particles, WIREs Nanomedicine Nanobiotechnol. 1 (2009) 299-310.

[47] B. Basly, D. Felder-Flesch, P. Perriat, C. Billotey, J. Taleb, G. Pourroya, S. BeginColin, Dendronized Iron Oxide Nanoparticles as Contrast Agents for MRI, vol. 46, 2010, pp. 985-987.

[48] E. Merbach, E. Tóth, The chemistry of contrast agents in medical magnetic resonance imaging, Wiley, 2001, p. 426.

[49] H. Duan, M. Kuang, X. Wang, Y.A. Wang, H. Mao, S. Nie, Reexamining the effects of particle size and surface chemistry on the magnetic properties of iron oxide nanocrystals: new insights into spin disorder and proton relaxivity, J. Phys. Chem. C 112 (2008) 8127-8131.

[50] A. Roch, R. Muller, P. Gillis, Theory of proton relaxation induced by superparamagnetic particles, J. Chem. Phys. 110 (10) (1999) 5403-5411.

[51] A. Carvalho, P.J. Sebastião, I. Fonseca, J. Matos, M. Clara Gonçalves, Silica and silica organically modified nanoparticles: water dynamics in complex systems, Microporous Mesoporous Mater. 217 (2015) 102-108. 